\title{
Systemic Rheumatoid Vasculitis: A Case Report
}

\author{
Dr. Bharat Veer Manchanda ${ }^{1}$,Dr Nishant Tayal ${ }^{2}$;Dr Saloni Mehra ${ }^{3}$; \\ Dr. Amit Rai ${ }^{4} ;$ Dr. Girish Dubey ${ }^{5}$;Dr.Sirobhi Sharma ${ }^{6}$ \\ ${ }^{I}$ (Asst. Professor; PG.Department Of Medicine, Subharti Medical College;Meerut, U.P.) \\ ${ }^{2}$ (JR III; PG.Department Of Medicine, Subharti Medical College;Meerut, U.P.) \\ ${ }^{3}$ (JR III; PG.Department Of Surgery, Subharti Medical College ;Meerut, U.P.) \\ ${ }^{4}$ (JR II; PG.Department Of Medicine, Subharti Medical College ;Meerut, U.P.) \\ ${ }_{5}^{5}$ (JR I; PG.Department Of Medicine, Subharti Medical College ;Meerut, U.P.) \\ ${ }^{6}$ (JR III; PG.Department Of Medicine, Subharti Medical College ;Meerut, U.P.)
}

\begin{abstract}
Rheumatoid Arthritis(RA)is a systemic inflammatory disease marked by severe symmetric polyarthritis. It is also characterised by a variety of extra-articular manifestations including fatigue lung involvement. Pericarditis., Peripheral neuropathy, vasculitis\& haematological abnormalities.Systemic Rheumatoid Vasculitis(SRV) though extremely rare is potentially the most severe form of of extra-articular manifestation. It is characterised by ischaemic inflammatory tissue damage affecting different systems particularly skin and peripheral Nervous system.Diagnosis of SRV requires a high degree of clinical suspicion and is difficult to diagnose. With emergence of DMARD's, particularly biological. The incidence of systemic SRV has decreased.It still occurs in high risk cases such as seropositive R.A. smoking, male gender, older age at diagnosis, Long-duration of disease, occurrence of Rheumatoid nodules and presence of HLADRB 1 \& HLAC3 Rheumatoid Vasculitis has a incidence of $<0.02 \%$ in General population. We report a case of elderly male with clinical picture of SRV
\end{abstract}

\section{Case Presentation}

Mr. Madan, $50 \mathrm{yr}$ old. Indian was admitted to hospital. He is a known case of Rheumatoid Arthritis for last 15 years,without any treatment since last 4 years.He was diagnosed as a case of R.A., according to 1987 revised criteria of American College of Rheumatology Presently over 4 criteria (i.e.morning stiffness; Arthritis of 3 or more joint areas; Symmetric Arthritis; Positive Rheumatic factors; Arthritis of hand joints) and scoring of > 6 according to new classification of RA 2010 ACR/EULAR criteria . (i.e. Joint involvement score of 3; serology score of 3; Acute Phase Reactant of 1, Duration of Symptoms1.) DAS severity score of 5.3 indicating sever disease activity.

9 years back, he c/o Numbness and Tingling sensations is b/l lower Limb (RT > Left) and minimal distal weakness in form of Planter \& dorsiflexor weakness and difficulty in walking,for this he was evaluated and EMG and NCV of all 4 Limbs revealed severe chronic asymmetrical polyneuropathies compatible with Mono-neuritis multiplex.

5 years ago pt developed complaints of gradually progressive pain is left leg which started from ankle and progressed to calf. He also had complaint of intermittent claudication. Pain was relieved after taking rest for some time. This was accompanied by blackish discolouration of left lower limb. It was diagnosed as a case of Gangrene with Septicaemia and subsequently left leg was amputated below knee.

3 years ago, He was admitted again with complaints of hard lumps in lateral aspect of Rt leg suggests of palpable purpura. This was also accompaid with ulceration and fever the lesions involved distal $2 / 3^{\text {rd }} \mathrm{Rt}$ leg.

1 year back he developed complaint of blurred vision and ocular dryness with irritative symptoms. For this he was evaluated and diagnosed as caseof pterygium and Keratoconjunctivitis sicca.

Presently patient presented with pain in chest radiating to left arm since 1 day. ECG of patient showed ST elevation of $>2 \mathrm{~mm}$ is left leads with positive, Trop T and elevated CPK-MB enzyme levels. He was diagnosed as a caseof anterior wall MI and was treated accordingly. Also he has c/o subcutaneous Oedema and localised loss of pigmentation with dilatation of capillary loops is Rt lower leg. This is accompanied with shallow ulcer irregular margins and minimal pain. Indicating vanous ulceration. alcoholic.

He also has h/o smoking (40 pack years) and has stopped smoking since 4 years. He is occasional

\section{Laboratory Tests}

CBC (by Calorimetric / electrical impedence method) Hb: $6.3 \mathrm{gm} / \mathrm{dL}$; Hct 21.9\% ; MCV $21.9 \mathrm{fl}$ (4050); $\mathrm{MCH} 19.8 \mathrm{pg}$ (27-32); MCHC $28.7 \mathrm{gm} / \mathrm{dL}$ (31.5-34.5); RDW 18.4\%.(11-14) P/C 4.82(1.5-4.5). WBC 9,500. Increased Rouleaux formation, Platelet Aggregates; Neutrophillia. Suggestive of chronic inflammatory 
state. ESR 144 (by Westergren method) S.Iron $20.0 \mathrm{mcg} / \mathrm{dl}(60-160 \mathrm{mg} / \mathrm{dL})$ Transferrin Saturation Index of $15 . \%(\operatorname{Ref} 20-25 \%)$.

KFT - Blood Urea 28.0 : S. Creatinine 0.6; Sodium 132 (Direct ISE); Potossium 4.1 (DirectISE) Calcium 8.1 (Arsenazo Dye Method) ; LFT. S.bilirubin Total 0.4, Direct 0.2; Indirect 0.2; Total Protein 6.1, Albumin 2.3; Globulin 3.8; SGOT 43.0, SGPT 31.0, S; Alkaline $\mathrm{PO}_{4}$ 98.0: Urine R/M Normal Study RA factor. 256 (Immunoturbidimetry) CRP 0.740 (Immunoturbidimetry) USG W/A revealed Right Renal calculus of 17mm. ANA Profile - Negative; Lupus Anti-coagulant Panel by Nephelometric and manual method was negative. $\mathrm{C}_{3} / \mathrm{C}_{4}$ levels were low. Anti $\mathrm{CCP}$ was 73 (Positive), $\mathrm{P}$ ANCA was positive. Viral markers were negative.

EMG \&NCV of all 4 Limbs was done and revealed severe chronic asymmetrical polyneuropathies suggestive of mononeuritis multiplex.Skin biopsy revealed patchy fibrinoid degeneration of vessel wall. The wall is infiltrated by neutrophills and some mononuculear inflammatory cells. Then features are consistent with leucocytoclastic vascultis.

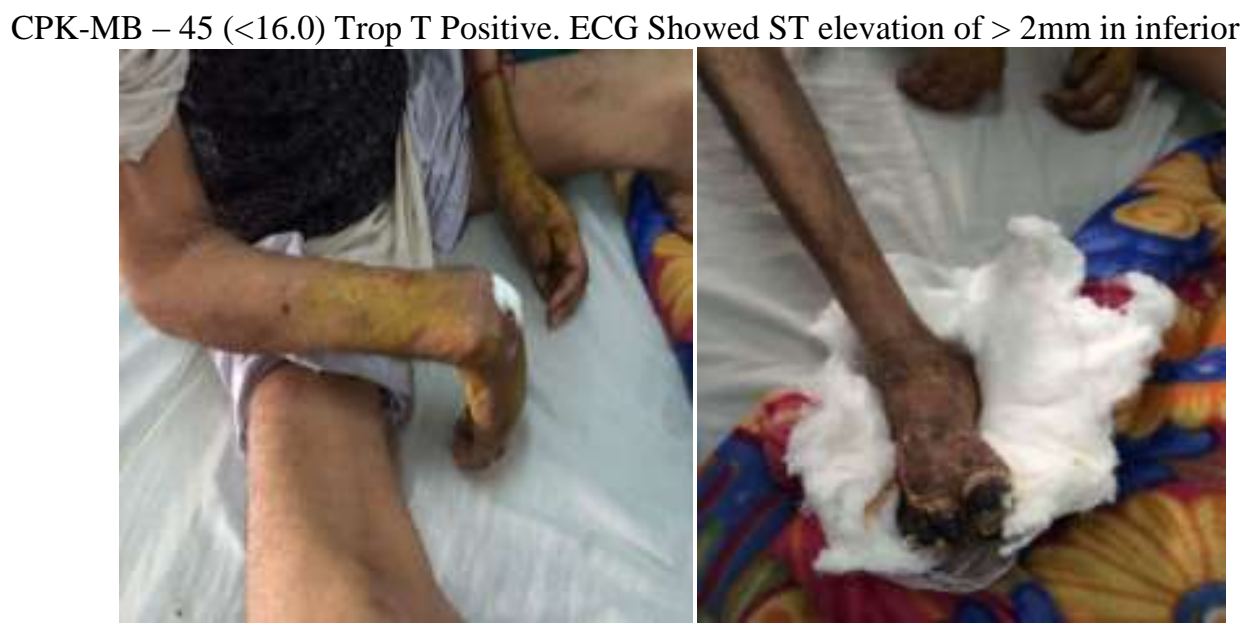

\section{Summary \& Discussion}

RA is a relatively common chronic inflammatory auto-immune disease that effect men 2 women of all ages. It more commonly effects women (men : women:: $1: 3$ ).SRV is a rare complication of R.A. Nowa days due to effective long term therapy which is available (DMARDS). The possibility of its occurrence should always be suspected if R.A. is associated with multisystem involvement notably the neuropathies and vasculitis. It requires a high degree of clinical suspicion.

Despite of reduced incidence the clinical course of the disease is unchanged and is usually associated with high rate of morbidity and mortality.Incidence of Rheumatoid Vasculities is more common in certain groups eg. Seropositive individuals, smoking, long disease duration age, male pre-pondrance and severity of the disease.

The physiology behind Rheumatoid Vasculities in still not completely understood but. It involves activation of complement cascade, immune-indicated inflammatory tissue iujury. Histologically it involves small medium sized vessels. The lesions involve entire length of the wall. There occurs perivascular fibrinoid necrosis and / OR leucocytoclasia. It may be associated with disruption of vascular structures and formation of intramural thrombi leading to impairment of local blood perfusion which may cause gangrene formation as happened with our case.

The clinical picture is based on symptoms of RA (eg Joint Pain, Early many stiffness, Rheumatoid nodules, Joint erosions), with or without fever;Skin involvement (80\%) which may be inform of superficial or deep skin ulcer, Gangrene, Petechiae or Purpura, necrotising lesions; Eye involvement as Episcleritis, Kerataconjunctivitis sicca;Involvement of peripheral nervous system in form of symmetrical distal sensory neuropathy lesions andmononeuritis multiplex. Other systems like lungs, Kidneys, GIT may be involved. If not recognised promptly \& treated it can lead to progressive organ dysfunction and fatal outcome.

SRV is defined using the Scott \& Bacon criterion i.e. Presence of one of the following features in a pt with RA : Mononeuntis multiplex, peripheral Gangrene. Acute necrotizing arteritis or deep cutaneous ulcers. Other causes of such lesions must be ruled out.

There is no established treatment protocol for SRV in mild cases prednisolone combined with methotrexate is used. In more severe cases, corticosteroides and cyclophosphamide have been used more effectively. Biological eg Infliximab and Rituximab have been used effectively especially in cases of refractory disease. 